\title{
P04-3-19 Poster session
}

\section{Periplocoside A ameliorated type II collagen-induced arthritis inmice via regulation of the balance of Th17/Treg cells}

\section{Jianping Zuo}

\author{
Shanghai Institute of Materia Medica, Chinese Academy of Sciences, China
}

Periplocoside A (PSA) has been extracted from the Chinese herbal medicine Periploca sepium Bge to treat rheumatoid arthritis (RA) via immune regulation.We previously found that PSA exhibits immunosuppressive activity both in vitro and in vivo. Balanced regulation of helper T 17 (Th17)/regulatory T (Treg) cells is the current therapeutic direction for the treatment of RA. The present study investigated the mechanismof PSA in treating collagen-induced arthritis (CIA). The therapeutic effects and potential pharmacological mechanisms of PSA were specifically clarified by examining its effects on CIA in DBA/1 mice. PSA administration significantly relieved the severity of the arthritis, and preventive administration of PSA reduced the incidence of arthritis in the mice with CIA and relieved joint damage in terms of morphology. PSA was also able to reduce the levels of anti-collagen II (CII) antibodies and pro-inflammatory cytokines in the serum. As a result, the proportion of Th17 cells decreased, and the proportion of Treg cells increased. A follow-up study of the ex vivo immunological reactions induced by a specific antigen found that PSA suppressed lymphocyte proliferation, inhibited the differentiation and reactivity of Th17 cells, and promoted the proportion of Treg cells among helper T cells. PSA also exhibited pharmacological effect in regulating the balance between Th17 and Treg cells in CIA through relevant signaling pathways. Thus, PSA played a specific role in CIA treatment. In particular, our results suggest that the therapeutic effects of PSA on RA are partially realized via the regulation of the balance of Th17/Treg cells. 